\title{
Controlled Generation of Coherent Matter Currents Using a Periodic Driving Field
}

\author{
C. E. Creffield and F. Sols \\ Dpto de Física de Materiales, Universidad Complutense de Madrid, E-28040, Madrid, Spain
}

(Received 13 February 2008; published 25 June 2008)

\begin{abstract}
We study the effect of a strong, oscillating driving field on the dynamics of ultracold bosons held in an optical lattice. Modeling the system as a Bose-Hubbard model, we show how the driving field can be used to produce and maintain a coherent atomic current by controlling the phase of the intersite tunneling processes. We investigate both the stroboscopic and time-averaged behavior using Floquet theory, and demonstrate that this procedure provides a stable and precise method of controlling coherent quantum systems.
\end{abstract}

DOI: 10.1103/PhysRevLett.100.250402

PACS numbers: 03.75.Lm, 03.65.Vf, 05.60.Gg

Introduction. - Recent experimental advances in the creation of Bose-Einstein condensates (BECs) from ultracold atomic gases have stimulated huge interest in investigating the coherent many-body dynamics of trapped bosons. By superposing counter-propagating laser beams, it is possible to impose extremely well-controlled lattice potentials on these systems. The precision and flexibility afforded by this both suggests their use as "quantum matter simulators" [1] for systems of interest from other areas of physics such as the integer quantum Hall effect [2], and also permits the clean observation of coherent lattice phenomena such as Bloch oscillations [3], the formation of repulsively bound pairs [4], and the Mott transition [5].

As well as their purely theoretical interest, these systems are also highly attractive candidates for applications such as quantum information processing due to their long coherence times. A powerful tool to control their dynamics is provided by the effect termed "coherent destruction of tunneling" [6], in which driving the system with an oscillating field has the effect of renormalizing the intersite tunneling amplitude. For certain parameters of the driving field, the tunneling can even be reduced to zero, and thus it has been proposed to use this effect to control the quantum phase transition between the superfluid and the Mott state $[7,8]$.

In this Letter, we show how an oscillating driving field can not only be used to control the amplitude of the tunneling, but also its phase. This permits the generation of a matter-current in analogy to the current induced in a conducting ring threaded by a magnetic flux. The creation of tunneling-phases has been studied before in BECs using either rotating lattices [9], or by using the atoms' internal degrees of freedom to mimic a fictitious magnetic field [10]. The scheme we propose is extremely simple in comparison, requiring only the controlled shaking of the optical lattice, which has already been demonstrated in experiment. It also reverses the normal role of CDT in a novel way, since the quantum interference effects which produce CDT are employed here to induce motion, rather than to suppress it. We study the effect over a range of interaction strengths, and find it to be present from weak interactions right up to the onset of the Mott state. A surprising feature is that while interactions do reduce the magnitude of the current, they do not introduce dephasing or dissipation as seen, for example, in Bloch oscillations [11], but instead render the current-generation more robust.

Model. - We consider a one-dimensional (1D) optical lattice, in which the atoms are confined to the lowest Bloch band. In this case, the system can be described very accurately [12] by the Bose-Hubbard (BH) model

$$
H_{\mathrm{BH}}=\sum_{\langle m, n\rangle}\left[-J a_{m}^{\dagger} a_{n}+\text { H.c. }\right]+\frac{U}{2} \sum_{m} n_{m}\left(n_{m}-1\right) .
$$

Here, $a_{m}\left(a_{m}^{\dagger}\right)$ are the standard boson destruction (creation) operators, $n_{m}=a_{m}^{\dagger} a_{m}$ is the number operator, and $U$ is the Hubbard-interaction between a pair of bosons occupying the same site. The tunneling amplitudes $J$ connect nearestneighbor sites $\langle m, n\rangle$, and we take $\hbar=1$. We now impose a time-dependent potential which rises linearly across the lattice

$$
H(t)=H_{\mathrm{BH}}+K(t) \sin (\omega t+\theta) \sum_{j} j n_{j},
$$

where $\omega$ is the frequency of the driving field, and $K(t)$ parameterizes its amplitude. Importantly, we include the phase of the driving field, $\theta$, as an additional control parameter. This form of potential can be produced by periodically phase-modulating one of the laser fields providing the optical lattice and has already been used in cold atom experiments [13] to induce CDT.

We begin by considering the case of a driving field of constant amplitude, $K(t)=K$. The Hamiltonian of the system, Eq. (2), is then periodic, with period $T=2 \pi / \omega$. Accordingly, we may use the Floquet theorem to write solutions of the time-dependent Schrödinger equation in the form $u(t)=\exp (-i \epsilon t) u(t)$, where $u(t)$ is a $T$-periodic function termed a Floquet state, and $\epsilon$ is called a quasienergy. In the high-frequency limit, where $\omega \gg(J, U)$ is the dominant energy scale of the problem, perturbative approximations to the Floquet states can be obtained by 
solving the Floquet equation for just the driving potential, and then including $H_{\mathrm{BH}}$ as a perturbation. In this case, following the procedure described in Refs. [14,15], the Floquet states are given to first-order by the eigenstates of an operator $\mathcal{H}(t)$ which is identical to $H_{\mathrm{BH}}$, but with periodically varying tunneling amplitudes given by

$$
J(t)=J \frac{1}{T} \int_{0}^{T} e^{ \pm i K F(\tau, t)} d \tau,
$$

where $F(\tau, t)=\int_{t}^{\tau} \sin \left(\omega t^{\prime}+\theta\right) d t^{\prime}$ is the phase accumulated over the interval $(t, \tau)$, and the $+(-)$ applies to forward (backward) hopping. If we now consider the system stroboscopically, that is, at discrete times $t=n T$ where $n$ is integer, the time dependence of the tunneling amplitudes disappears, and the system is effectively governed by a static Hamiltonian $\mathcal{H}(0)$, with the Floquet states $u_{j}(0)$ playing the role of energy eigenvectors. Simplifying Eq. (3) then reveals that the action of the driving field is to renormalize the tunneling amplitudes as

$$
J_{\text {eff }}=J e^{ \pm i(K / \omega) \cos \theta} \mathcal{J}_{0}(K / \omega),
$$

where $\mathcal{J}_{0}$ is the zeroth Bessel function of the first kind. For $\theta=\pi / 2$ (cosinusoidal driving), this result reproduces the familiar Bessel function renormalization [7,8] of the tunneling. An unanticipated result, however, is that the hopping in general acquires a nonzero phase, which is maximum for $\theta=0$ (sinusoidal driving).

It may appear surprising that $\theta$ can induce a nontrivial phase, as changing $\theta$ is equivalent merely to shifting the time origin. In practice, however, the driving field must be turned on at a certain time, which thereby does pick out a specific value for the phase of the driving field. Since the system is completely coherent, the effect of this initial condition is not lost during the subsequent time-evolution, and thus the driving phase $\theta$ can produce different physical results. The central result of our work is that if $K$ is increased from zero sufficiently slowly, the Floquet states of the system are able to adiabatically follow [16], and thereby acquire the $K$-dependent phase $\phi=K(t) \cos \theta / \omega$. As a result, a given initial state can be transformed into a current-carrying state by slowly ramping the driving potential from zero to the value that gives the desired hopping-phase.

Results. - To verify these results, we study the behavior of an $N$-site BH system by numerically propagating the many-particle wave function under the time-dependent Hamiltonian (2). We focus on the case of commensurate filling, where the number of bosons is equal to $N$, so that in the limit of large $U$, a well-defined Mott state exists. The rapid increase in the dimension of the Hilbert space means that we could only consider systems of up to $N=10$, but examining the results as $N$ is increased reveals that the behavior we find is quite insensitive to lattice size. To probe the behavior of the system, we measure the singleparticle momentum distribution

$$
\rho(p, t)=\frac{1}{N} \sum_{m, n}^{N} e^{i(m-n) p}\left\langle\psi(t)\left|a_{m}^{\dagger} a_{n}\right| \psi(t)\right\rangle .
$$

This quantity is remarkably size-independent [17], allowing results from small lattice systems to be reliably extrapolated to the thermodynamic limit. It can be observed directly in experiment by time-of-flight absorption imaging, and conveniently indicates whether the system is in the superfluid or Mott-insulator regime [5]. When interactions are weak $(U \ll J)$, bosons are delocalized over the lattice in a superfluid state, and the system possesses long-range phase coherence. Consequently, $\rho(p)$ is sharply peaked, as shown in Fig. 1. As the interaction is increased, the peaks broaden and reduce in height, indicating that the bosons become progressively more localized on the lattice sites. For sufficiently large values of $U / J$, the atoms localize completely to form the Mott state, for which the momentum distribution is completely flat. In $1 \mathrm{D}$, this phase transition is quite soft, giving a large range of $U$ over which $\rho(p)$ is peaked.

We begin by considering the case of an intermediate interaction strength, $U=8 \mathrm{~J}$. To place the system in the high-frequency regime, we set $\omega=30 \mathrm{~J}$ and use a sinusoidal driving field $(\theta=0)$. The precise form in which $K(t)$ is increased from zero is not important as long as it satisfies the adiabaticity constraint, and for simplicity, we consider a linear ramp $K(t)=K_{0} t$. As noted earlier, we will evaluate all physical quantities stroboscopically at times $t=n T$. The system is initialized in its ground-state, and in Fig. 2(a), we plot the expectation value of the lattice current, $I=2 J \operatorname{Im}\left\langle a_{m} a_{n}^{\dagger}\right\rangle$, as a function of the driving amplitude. For $K(t)=0$, the current is zero due to the

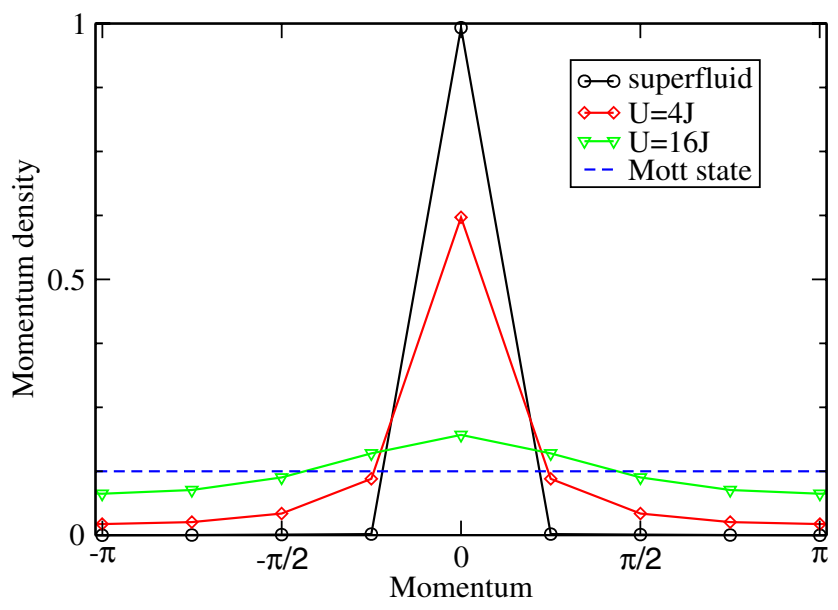

FIG. 1 (color online). Normalized momentum density $\rho(p) / N$ for the first Brillouin zone of an 8-site lattice holding eight bosons. For a perfect superfluid, the momentum density is sharply peaked, showing the presence of long-range coherence. As $U$ is increased, the peak flattens and broadens, until for $U=$ $16 \mathrm{~J}$, the distribution is almost flat, indicating the proximity to the formation of a Mott insulator. 

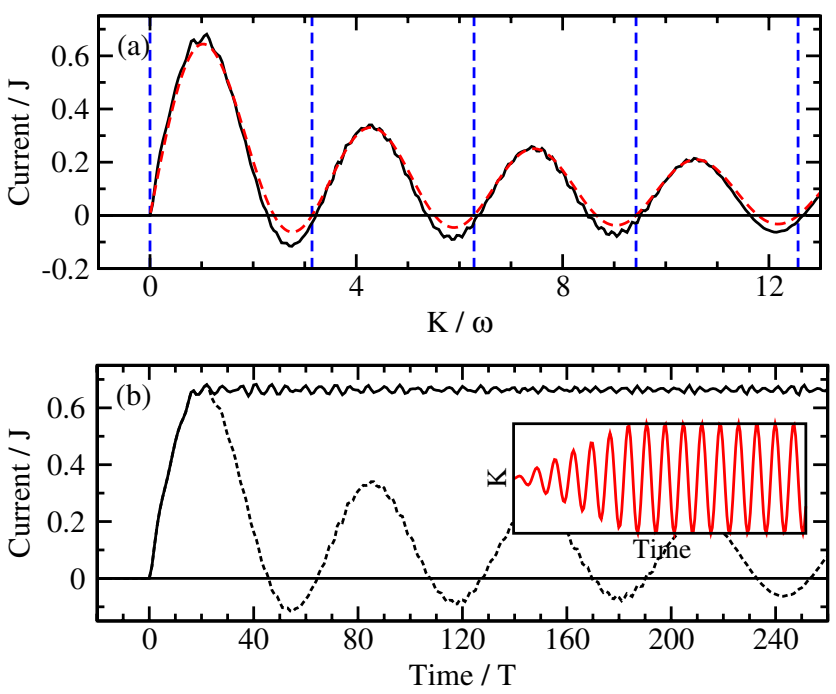

FIG. 2 (color online). Current induced by a linearly ramped driving field $K(t)=K_{0} t$, where $K_{0}=0.05 T^{-1}$, in an 8 -site system with interaction strength $U=8 J$. The driving is sinusoidal $(\theta=0)$. (a) The induced current (solid line), shows a decaying oscillatory behavior, described well by Eq. (6) (dashed line). As well as at multiples of $\pi$, marked by the vertical lines, zeros of the current also occur when $\mathcal{J}_{0}(K / \omega)=0$ due to CDT. (b) As above, the dashed line indicates the current produced by a continuously ramped field. Holding $K(t)$ constant after a certain time (shown schematically in the inset) keeps the current at a constant level; the solid curve shows the effect of ramping the field until $t=21 T$, which gives the maximum current $I_{0}$.

symmetry of the momentum distribution. As $K$ is increased, however, the peak in $\rho(p)$ is displaced from the center of the Brillouin zone due to the induced hoppingphase $\phi, \rho(p) \rightarrow \rho(p+\phi)$. As a result, $I$ becomes nonzero due to the imbalance between the left- and rightmoving momentum components.

Following its initial increase, $I$ displays a damped oscillatory dependence on $K / \omega$. To interpret this behavior, we show the corresponding response of the momentum density in Fig. 3. As expected, the initial effect of the ramping potential is simply to shift the locations of the peaks in the momentum distribution by inducing the hopping phase. As the peaks shift, however, their amplitude is reduced by the Bessel function renormalization of the hopping amplitude (4). As $K / \omega \rightarrow 2.4048$, the first zero of $\mathcal{J}_{0}$, the effective hopping vanishes, and the system thus makes a transition to the Mott state [7], and the momentum density flattens. As $K$ is increased further, peaks reappear in $\rho(p)$, but their location is discretely shifted. This occurs because $J_{\text {eff }}$ has become negative; writing it as $J_{\text {eff }}=\left|J_{\text {eff }}\right| \exp (i \pi)$ clearly indicates that the peaks in the momentum density will be displaced by $\pi$. Predicted in Ref. [18], this shift has recently been experimentally observed for a weakly interacting $(U \simeq 0.1 J)$ system in Ref. [13]. The intricate behavior of $I$ in Fig. 2(a) thus arises from a combination of the

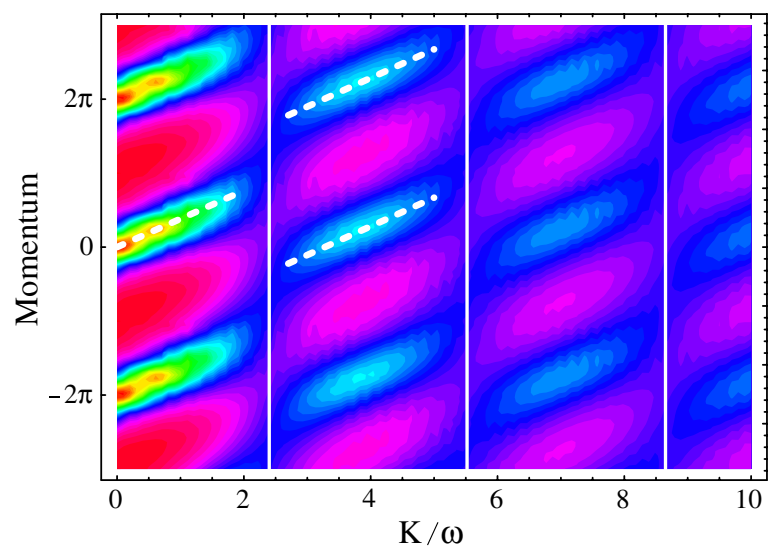

FIG. 3 (color online). Momentum distribution for an 8-site system $(U=8 J)$ under sinusoidal driving with a linearly ramped amplitude. To guide the eye, three Brillouin zones are plotted, and dashed lines indicate the evolution of the central peak. The vertical lines mark the zeros of $\mathcal{J}_{0}$. As $K$ increases, the peaks steadily shift in momentum due to the induced hopping phase, and their amplitude reduces according to the Bessel function $\mathcal{J}_{0}(K / \omega)$. At $K / \omega=2.4048$, the Bessel function approaches zero, and the system becomes a Mott insulator with a flat distribution. Increasing $K$ further causes $J_{\text {eff }}$ to change sign, and the peaks reappear with a shift of $\pi$ (see text). This pattern then repeats.

shifting location of the peaks, together with many-particle effects arising from the competition between $J_{\text {eff }}$ and $U$. The roughness visible in the current arises from departures from adiabaticity in the driving. As the ramping rate is decreased, and so approximates adiabatic evolution more closely, this roughness is progressively eliminated.

For strong interactions, the ground state of the system consists approximately of the Mott state, $|11111 \ldots\rangle$, with a small admixture of excited states. The dominant dynamical processes will be nearest-neighbor tunneling between the Mott state and "particle-hole" states, separated from the Mott state by an energy gap of $\sim U$, where one site is doubly occupied and one site is empty (e.g., |12011 ...). Including only these processes, we can obtain an approximate form for the induced current

$$
I \simeq 2 J|\alpha|^{2} \sin (K \cos \theta / \omega) \mathcal{J}_{0}(K / \omega),
$$

where $|\alpha|^{2}$ is the weight of the particle-hole states in the interacting ground state. For large $U,|\alpha|^{2}$ decays as $\sim U^{-1}$ as the ground state converges toward the Mott state. Using $\alpha$ as a fitting parameter, we show in Fig. 2(a) that this expression indeed provides an excellent description of the current. Equation (6) reveals the two distinct sources for the zeros of current: (i) when $K / \omega=n \pi$, the momentum density is symmetrically peaked at the center of the Brillouin zone, and the positive and negative currents cancel, (ii) when the Bessel function becomes zero, $J_{\text {eff }}$ is suppressed, and so the tunneling itself is quenched. 




FIG. 4 (color online). The maximum current, $I_{0}$, induced in an 8-site system depends on the phase of the driving field, $\theta$. In all cases, $I_{0}$ is maximized for sinusoidal driving and is zero for the cosinusoidal case. Solid lines plot the dependence $\gamma \sin (K \cos \theta / \omega)$, where $\gamma$ is a fitting parameter, and show excellent agreement with Eq. (6). As $U$ is increased, $I_{0}$ reduces, but even for $U=16 \mathrm{~J}$, the induced current is significant.

An important consequence of the interaction is that larger values of $U$ confer increased stability during the ramping process. For weak interactions, only extremely slow ramping can be used, or the system will be excited from its instantaneous ground state, and control of the coherent current will be lost. When $U$ is large, however, the resulting energy gap isolates the ground state from the rest of the spectrum and makes the adiabatic condition easier to attain, thereby allowing more rapid ramping to be used.

Differentiating Eq. (6) reveals that the maximum current, $I_{0}$, occurs for $K / \omega \simeq 1.0311$. In Fig. 2(b), we show the effect of ramping the value of $K(t)$ up to this value, and then keeping it fixed (see inset), which maintains the induced current at its final value. The magnitude of the current depends only on the final value of $K / \omega$, and so by regulating this value, any desired value of current within the range $\pm I_{0}$ can be generated. In Fig. 4 we show the dependence of $I_{0}$ on the phase of the driving $\theta$ for several different values of $U$. All the curves show the $\sin (K \cos \theta / \omega)$ dependence expected from Eq. (6), and the magnitude of the current remains significant even for large interaction strengths near the onset of the Mott transition. While smaller values of $U$ allow larger currents to be induced, slower ramping rates must then be used, giving a trade-off between the two effects in experimental implementations.

Conclusions. - We have described a means of inducing a coherent atomic current by adiabatically controlling the renormalization of the intersite tunneling. The presence of interactions both stabilizes this mechanism, and also introduces novel strong-correlation effects. To reveal this effect, we have employed a stroboscopic measurement scheme; this implies that in experiment, measurements must be made at well-controlled intervals and be sufficiently rapid to reflect the system's instantaneous state. For typical cold atom systems, this would require temporal control of the order of milliseconds, which should be easily achievable [13]. If the measurements have insufficient time resolution, it would then be appropriate to consider the time average of Eq. (3), which yields the result that $\left\langle J_{\text {eff }}\right\rangle=$ $J \mathcal{J}_{0}^{2}(K / \omega)$, and thus the hopping-phase vanishes and the effective hopping is proportional to the square of the Bessel function. For a tilted lattice, it can be shown that the tunneling is renormalized as $\mathcal{J}_{m}^{2}(K / \omega)$, where $m \omega$ is the energy difference between adjacent sites. Interestingly, such a dependence has been recently observed in [19] for $m=1,2$. While we have focused on the case of bosonic atoms, this mechanism should be equally applicable to cold fermionic atoms or electronic systems, provided that they possess the required coherence properties.

We thank Martin Holthaus for stimulating discussions. This work was funded by the MEC (Spain) through Grant Nos. FIS2004-05120 and FIS2007-65723. C. E. C. was supported by a Ramón y Cajal Fellowship.

[1] D. Jaksch and P. Zoller, Ann. Phys. (N.Y.) 315, 52 (2005).

[2] B. Paredes, P. Zoller, and J.I. Cirac, Phys. Rev. A 66, 033609 (2002).

[3] O. Morsch, J. H. Müller, M. Cristiani, D. Ciampini, and E. Arimondo, Phys. Rev. Lett. 87, 140402 (2001).

[4] K. Winkler, G. Thalhammer, F. Lang, R. Grimm, J. Hecker Denschlag, A. J. Daley, A. Kantian, H. P. Buchler, and P. Zoller, Nature (London) 441, 853 (2006).

[5] M. Greiner, O. Mandel, T. Esslinger, T. W. Hänsch, and I. Bloch, Nature (London) 415, 39 (2002).

[6] F. Grossmann, T. Dittrich, P. Jung, and P. Hänggi, Phys. Rev. Lett. 67, 516 (1991).

[7] A. Eckardt, C. Weiss, and M. Holthaus, Phys. Rev. Lett. 95, 260404 (2005).

[8] C. E. Creffield and T. S. Monteiro, Phys. Rev. Lett. 96, 210403 (2006).

[9] V. Schweikhard, I. Coddington, P. Engels, V.P. Mogendorff, and E. A. Cornell, Phys. Rev. Lett. 92, 040404 (2004).

[10] D. Jaksch and P. Zoller, New J. Phys. 5, 56 (2003).

[11] A. Buchleitner and A. R. Kolovsky, Phys. Rev. Lett. 91, 253002 (2003).

[12] D. Jaksch, C. Bruder, J. I. Cirac, C. W. Gardiner, and P. Zoller, Phys. Rev. Lett. 81, 3108 (1998).

[13] H. Lignier, C. Sias, D. Ciampini, Y. Singh, A. Zenesini, O. Morsch, and E. Arimondo, Phys. Rev. Lett. 99, 220403 (2007).

[14] C. E. Creffield, Phys. Rev. B 67, 165301 (2003).

[15] S. Kohler, J. Lehmann, and P. Hänggi, Phys. Rep. 406, 379 (2005).

[16] K. Drese and M. Holthaus, Eur. Phys. J. D 5, 119 (1999); A. Eckardt and M. Holthaus, J. Phys.: Conf. Ser. 99, 012007 (2008).

[17] D.-S. Lühmann, K. Bongs, K. Sengstock, and D. Pfannkuche, Phys. Rev. A 77, 023620 (2008).

[18] A. Eckardt and M. Holthaus, Europhys. Lett. 80, 50004 (2007).

[19] C. Sias, H. Lignier, Y. P. Singh, A. Zenesini, D. Ciampini, O. Morsch, and E. Arimondo, Phys. Rev. Lett. 100, 040404 (2008). 